Georgian Mathematical Journal

1(1994), No. 2, 141-150

\title{
ON THE CORRECT FORMULATION OF A MULTIDIMENSIONAL PROBLEM FOR STRICTLY HYPERBOLIC EQUATIONS OF HIGHER ORDER
}

\author{
S. KHARIBEGASHVILI
}

\begin{abstract}
A theorem of the unique solvability of the first boundary value problem in the Sobolev weighted spaces is proved for higherorder strictly hyperbolic systems in the conic domain with special orientation.
\end{abstract}

In the space $R^{n}, n>2$, let us consider a strictly hyperbolic equation of the form

$$
p(x, \partial) u(x)=f(x),
$$

where $\partial=\left(\partial_{1}, \ldots, \partial_{n}\right), \partial_{j}=\partial / \partial x_{j}, p(x, \xi)$ is a real polynomial of order $2 m, m>1$, with respect to $\xi=\left(\xi_{1}, \ldots, \xi_{n}\right), f$ is the known function and $u$ is the unknown function. It is assumed that in equation (1) the coefficients at higher derivatives are constant and the other coefficients are finite and infinitely differentiable in $R^{n}$.

Let $D$ be a conic domain in $R^{n}$, i.e., $D$ together with a point $x \in D$ contains the entire beam $t x, 0<t<\infty$. Denote by $\Gamma$ the cone $\partial D$. It is assumed that $D$ is homeomorphic onto the conic domain $x_{1}^{2}+\cdots+x_{n-1}^{2}-$ $x_{n}^{2}<0, x_{n}>0$ and $\Gamma^{\prime}=\Gamma \backslash O$ is a connected $(n-1)$-dimensional manifold of the class $C^{\infty}$, where $O$ is the vertex of the cone $\Gamma$.

Consider the problem: Find in the domain $D$ the solution $u(x)$ of equation (1) by the boundary conditions

$$
\left.\frac{\partial^{i} u}{\partial \nu^{i}}\right|_{\Gamma^{\prime}}=g_{i}, \quad i=0, \ldots, m-1,
$$

where $\nu=\nu(x)$ is the outward normal to $\Gamma^{\prime}$ at a point $x \in \Gamma^{\prime}$, and $g_{i}$, $i=0, \ldots, m-1$, are the known real functions.

Note that the problem (1), (2) is considered in [1-6] for a hyperbolictype equation of second order when $\Gamma$ is a characteristic conoid. In [7] this

1991 Mathematics Subject Classification. 35L35. 
problem is considered for a wave equation when the conic surface $\Gamma$ is not characteristic at any point and has a time-type orientaton. A multidimensional analogue of the problem is treated in [8-10] for the case when one part of the cone $\Gamma$ is characteristic and the other part is a time-type hyperplane. Other multidimensional analogues of the Goursat problem for hyperbolic systems of first and second order are investigated in [11-15].

In this paper we consider the question whether the problem (1), (2) can be correctly formulated in special weighted spaces $W_{\alpha}^{k}(D)$ when the cone $\Gamma$ is assumed not to be characteristic but having a quite definite orientation.

Denote by $p_{0}(\xi)$ the characteristic polynomial of the equation (1), i.e., the higher homogeneous part of the polynomial $p(x, \xi)$. The strict hyperbolicity of the equation (1) implies the existence of a vector $\zeta \in R^{n}$ such that the straight line $\xi=\lambda \zeta+\eta$, where $\eta \in R^{n}$ is an arbitrarily chosen vector not parallel to $\zeta$ and $\lambda$ is the real parameter, intersects the cone of normals $K: p_{0}(\xi)=0$ of the equation (1) at $2 m$ different real points. In other words, the equation $p_{0}(\lambda \zeta+\eta)=0$ with respect to $\lambda$ has $2 m$ different real roots. The vector $\zeta$ is called a spatial-type normal. As is well-known, a set of all spatial-type normals form two connected centrally-symmetric convex conic domains whose boundaries $K_{1}$ and $K_{2 m}$ give the internal cavity of the cone of normals $K$ [3]. The surface $S \subset R^{n}$ is called characteristic at a point $x \in S$ if the normal to $S$ at the point $x$ belongs to the cone $K$.

Let the vector $\zeta$ be a spatial-type normal and the vector $\eta \neq 0$ change in the plane orthogonal to $\zeta$. Then for $\lambda$ the roots of the characteristic polynomial $p_{0}(\lambda \zeta+\eta)$ can renumerated so that $\lambda_{2 m}(\eta)<\lambda_{2 m-1}(\eta)<\cdots<$ $\lambda_{1}(\eta)$. It is obvious that the vectors $\lambda_{i}(\eta) \zeta+\eta$ cover the cavities $K_{i}$ of $K$ when the $\eta$ changes on the plane othogonal to $\zeta$. Since $\lambda_{m-j}(\eta)=$ $-\lambda_{m+j+1}(-\eta), 0 \leq j \leq m-1$, the cones $K_{m-j}$ and $K_{m+j+1}$ are centrally symmetric with respect to the point $(0, \ldots, 0)$. As is well-known, by the bicharacteristics of the equation (1) we understand straight beams whose orthogonal planes are tangential planes to one of the cavities $K_{i}$ at the point different from the vertex.

Assume that there exists a plane $\pi_{0}$ such that $\pi_{0} \cap K_{m}=\{(0, \ldots, 0)\}$. This means that the cones $K_{1}, \ldots, K_{m}$ are located on one side of $\pi_{0}$ and the cones $K_{m+1}, \ldots, K_{2 m}$ on the other. Set $K_{i}^{*}=\cap_{\eta \in K_{i}}\left\{\xi \in R^{n}: \xi \cdot \eta<0\right\}$, where $\xi \cdot \eta$ is the scalar product of $\xi$ and $\eta$. Since $\pi_{0} \cap K_{m}=\{(0, \ldots, 0)\}$, $K_{i}^{*}$ is a conic domain and $K_{m}^{*} \subset K_{m-1}^{*} \subset \cdots \subset K_{1}^{*}, K_{m+1}^{*} \subset K_{m+2}^{*} \subset \cdots \subset$ $K_{2 m}^{*}$. It is easy to verify that $\partial\left(K_{i}^{*}\right)$ is a convex cone whose generatrices are bicharacteristics; note that in this case none of the bicharacteristics of the equation (1) comes from the point $(0, \ldots, 0)$ into the cone $\partial\left(K_{m}^{*}\right)$ or $\partial\left(K_{m+1}^{*}\right)[3]$.

Let us consider

Condition 1. The surface $\Gamma^{\prime}$ is characteristic at none of its points and 
each generatrix of the cone $\Gamma$ has the direction of a spatial-type normal; moreover, $\Gamma \subset K_{m}^{*} \cup 0$ or $\Gamma \subset K_{m+1}^{*} \cup 0$.

Denote by $W_{\alpha}^{k}(D), k \geq 2 m,-\infty<\alpha<\infty$, the functional space with the norm [16]

$$
\|u\|_{W_{\alpha}^{k}(D)}^{2}=\sum_{i=0}^{k} \int_{D} r^{-2 \alpha-2(k-i)}\left\|\frac{\partial^{i} u}{\partial x^{i}}\right\|^{2} d x
$$

where

$$
r=\left(x_{1}^{2}+\cdots+x_{n}^{2}\right)^{\frac{1}{2}}, \quad \frac{\partial^{i} u}{\partial x^{i}}=\frac{\partial^{i} u}{\partial x_{1}^{i_{1}} \cdots \partial x_{n}^{i_{n}}}, \quad i=i_{1}+\cdots+i_{n} .
$$

The space $W_{\alpha}^{k}(\Gamma)$ is defined in a similar manner.

Consider the space

$$
V=W_{\alpha-1}^{k+1-2 m}(D) \times \prod_{i=0}^{m-1} W_{\alpha-\frac{1}{2}}^{k-i}(\Gamma) .
$$

Assume that to the problem (1), (2) there corresponds the unbounded operator

$$
T: W_{\alpha}^{k}(D) \rightarrow V
$$

with the domain of definition $\Omega_{T}=W_{\alpha-1}^{k+1}(D) \subset W_{\alpha}^{k}(D)$, acting by the formula

$$
T u=\left(p(x, \partial) u,\left.u\right|_{\Gamma^{\prime}}, \ldots,\left.\frac{\partial^{i} u}{\partial \nu^{i}}\right|_{\Gamma^{\prime}}, \ldots,\left.\frac{\partial^{m-1} u}{\partial \nu^{m-1}}\right|_{\Gamma^{\prime}}\right), \quad u \in \Omega_{T} .
$$

It is obvious that the operator $T$ admits the closure $\bar{T}$.

The function $u$ is called a strong solution of the problem (1), (2) of the class $W_{\alpha}^{k}(D)$ if $u \in \Omega_{\bar{T}}, \bar{T} u=\left(f, g_{0}, \ldots, g_{m-1}\right) \in V$, which is equivalent to the existence of a sequence $u_{i} \in \Omega_{T}=W_{\alpha-1}^{k+1}(D)$ such that $u_{i} \rightarrow u$ in $W_{\alpha}^{k}(D)$ and $\left(p(x, \partial) u_{i},\left.u_{i}\right|_{\Gamma^{\prime}}, \ldots,\left.\frac{\partial^{m-1} u_{i}}{\partial \nu^{m-1}}\right|_{\Gamma^{\prime}}\right) \rightarrow\left(f, g_{0}, \ldots, g_{m-1}\right)$ in $V$.

Below, by a solution of the problem (1), (2) of the class $W_{\alpha}^{k}(D)$ we will mean a strong solution of this problem in the sense as indicated above.

We will prove

Theorem. Let condition 1 be fulfilled. Then there exists a real number $\alpha_{0}=\alpha_{0}(k)>0$ such that for $\alpha \geq \alpha_{0}$ the problem (1), (2) is uniquely solvable in the class $W_{\alpha}^{k}(D)$ for any $f \in W_{\alpha-1}^{k+2 m}(D), g_{i} \in W_{\alpha-\frac{1}{2}}^{k-i}(\Gamma)$, $i=0, \ldots, m-1$, and to obtain the solution $u$ we have the estimate

$$
\|u\|_{W_{\alpha}^{k}(D)} \leq c\left(\sum_{i=1}^{m-1}\left\|g_{i}\right\|_{W_{\alpha-\frac{1}{2}}^{k-i}(\Gamma)}+\|f\|_{W_{\alpha-1}^{k+1-2 m}(D)}\right),
$$


where $c$ is a positive constant not depending on $f, g_{i}, i=0, \ldots, m-1$.

Proof. First we will show that the corollaries of condition 1 are the following conditions: Take any point $P \in \Gamma^{\prime}$ and choose a Cartesian system $x_{1}^{0}, \ldots, x_{n}^{0}$ connected with this point and having vertex at $P$ such that the $x_{n}^{0}$-axis is directed along the generatrix of $\Gamma$ passing through $P$ and the $x_{n-1}^{0}$-axis is directed along the inward normal to $\Gamma$ at this point.

Condition 2. The surface $\Gamma^{\prime}$ is characteristic at none of its points. Each generatrix of the cone $\Gamma$ has the direction of a spatial-type normal, and exactly $m$ characteristic planes of equation (1) pass through the $(n-2)$ dimensional plane $x_{n}^{0}=x_{n-1}^{0}=0$ connected with an arbitrary point $P \in \Gamma^{\prime}$ into the angle $x_{n}^{0}>0, x_{n-1}^{0}>0$.

Denote by $\widetilde{p_{0}}(\xi)$ the characteristic polynomial of the equation (1) written in terms of the coordinate system $x_{1}^{0}, \ldots, x_{n}^{0}$, connected with an arbitrarily chosen point $P \in \Gamma^{\prime}$.

Condition 3. The surface $\Gamma^{\prime}$ is characteristic at none of its point. Each generatrix of the cone $\Gamma$ has the direction of a spatial-type normal and for $\operatorname{Re} s>0$ the number of roots $\lambda_{j}\left(\xi_{1}, \ldots, \xi_{n-2}, s\right)$, if we take into account the multiplicity of the polynomial $\widetilde{p_{0}}\left(i \xi_{1}, \ldots, i \xi_{n-2}, \lambda, s\right)$ with $\operatorname{Re} \lambda_{j}<0$, is equal to $m, i=\sqrt{-1}$.

When condition 3 is fulfilled, the polynomial $\widetilde{p_{0}}\left(i \xi_{1}, \ldots, i \xi_{n-2}, \lambda, s\right)$ can be written as the product $\Delta_{-}(\lambda) \Delta_{+}(\lambda)$, where for $\operatorname{Re} s>0$ the roots of the polynomials $\Delta_{-}(\lambda)$ and $\Delta_{+}(\lambda)$ lie, respectively, to the left and to the right of the imaginary axis, while the coefficients are continuous for $s, \operatorname{Re} s \geq$ $0,\left(\xi_{1}, \ldots, \xi_{n-2}\right) \in R^{n-2}, \xi_{1}^{2}+\cdots+\xi_{n-2}^{2}+|s|^{2}=1[17]$. On the left side of the boundary conditions (2) to the differential operator $b_{j}(x, \partial), 0 \leq$ $j \leq m-1$, written in terms of the coordinate system $x_{1}^{0}, \ldots, x_{n}^{0}$ connected with the point $P \in \Gamma^{\prime}$, there corresponds the characteristic polynomial $b_{j}(\xi)=\xi_{n-1}^{j}$. Therefore, since the degree of the polynomial $\Delta_{-}(\lambda)$ is equal to $m$, the following condition will be fulfilled:

Condition 4. For any point $P \in \Gamma^{\prime}$ and any $s, \operatorname{Re} s \geq 0$, and $\left(\xi_{1}, \ldots, \xi_{n-2}\right) \in$ $R^{n-2}$ such that $\xi_{1}^{2}+\cdot+\xi_{n-2}^{2}+|s|^{2}=1$, the polynomials $b_{j}\left(i \xi_{1}, \ldots, i \xi_{n-2}, \lambda, s\right)=$ $\lambda^{j}, j=0, \ldots, m-1$, are linearly independent, like the polynomials of $\lambda$ modulo $\Delta_{-}(\lambda)$.

We will now show that condition 1 implies condition 2, while the latter implies condition 3. Let us consider the case $\Gamma \subset K_{m+1}^{*} \cup O$. The second case $\Gamma \subset K_{m}^{*} \cup O$ is treated similarly.

Let $P \in \Gamma^{\prime}$ and $x_{1}^{0}, \ldots, x_{n}^{0}$ be the coordinate system connected with this point. Since the generatrix $\gamma$ of the cone $\Gamma$ passing through this point is a spatial-type normal, the plane $x_{n}^{0}=0$ passing through the point $P$ is 
a spatial-type plane. Denote by $K_{j}^{\wedge}$ the boundary of the convex shell of the set $K_{j}$ and by $K_{j}^{\perp}$ the set which is the union of all bicharacteristics corresponding to the cone $K_{j}$ and coming out of the point $O$ along the outward normal to $K_{j}, 1 \leq j \leq 2 m$. It is obvious that $\left(K_{j}^{\wedge}\right)^{*}=K_{j}^{*}$, $\partial\left(K_{j}^{*}\right)=\left(K_{j}^{\wedge}\right)^{\perp}$. We will show that the plane $\pi_{1}$, parallel to the plane $x_{n}^{0}=0$ and passing through the point $(0, \ldots, 0)$, is the plane of support to the cone $K_{m}^{\wedge}$ at the point $(0, \ldots, 0)$. Indeed, it is obvious that the plane $N \cdot \xi=0, N \in R^{n} \backslash(0, \ldots, 0), \xi \in R^{n}$ is the plane of support to $K_{m}^{\wedge}$ at the point $(0, \ldots, 0)$ iff the normal vector $N$ to this plane taken with the sign + or - belongs to the conic domain closure $\left(K_{m}^{\wedge}\right)^{*}=K_{m}^{*}$. Now it remains for us to note that the conic domains $K_{m}^{*}$ and $K_{m+1}^{*}$ are centrally symmetric with respect to the point $(0, \ldots, 0)$, and the generatrix $\Gamma$ passing through the point $P$ is perpendicular to the plane $\pi_{1}$ and, by the condition, belongs to the set $K_{m+1}^{*} \cup O$. Since $x_{n}^{0}=0$ is a spatial-type plane, the two-dimensional plane $\sigma: x_{1}^{0}=\cdots=x_{n-2}^{0}=0$ passing through the generatrix $\gamma$ which is directed along the spatial-type normal intersects the cone of normals $K_{p}$ of equation (1) with vertex at the point $P$ by $2 m$ different real straight lines [3]. The planes orthogonal to these straight lines and passing through the $(n-2)$-dimensional plane $x_{n}^{0}=x_{n-1}^{0}=0$ give all $2 m$ characteric planes passing through the $(n-2)$-dimensional plane $x_{n}^{0}=x_{n-1}^{0}=0$. The straight lines $x_{n}^{0}=0$ and $x_{n-1}^{0}=0$ divide the two-dimensional plane $\sigma$ into four right angles

$$
\begin{aligned}
& \sigma_{1}: x_{n-1}^{0}>0, x_{n}^{0}>0 ; \quad \sigma_{2}: x_{n-1}^{0}<0, x_{n}^{0}>0 \\
& \sigma_{3}: x_{n-1}^{0}<0, x_{n}^{0}<0 ; \quad \sigma_{4}: x_{n-1}^{0}>0, x_{n}^{0}<0 .
\end{aligned}
$$

One can readily see that exactly $m$ characteristic planes of equation (1) pass through the $(n-2)$-dimensional plane $x_{n}^{0}=x_{n-1}^{0}=0$ into the angle $x_{n}^{0}>0$, $x_{n-1}^{0}$ iff exactly $m$ straight lines from the intersection of $\sigma_{4}$ with the twodimensional plane $\sigma$ pass into the angle $K_{P}$. The latter fact really occurs, since: 1$)$ the plane $x_{n}^{0}=0$ is the plane of support to $K_{m}^{\wedge}$ and therefore to all $\left.K_{1}, \ldots, K_{2 m} ; 2\right)$ the planes $x_{n}^{0}=0, x_{n-1}^{0}=0$ are not characteristic because the generatrices of $\Gamma$ have a spatial-type direction and $\Gamma$ is not characteristic at the point $P$.

Now it will be shown that condition 2 implies condition 3. By virtue of condition 2 the plane $x_{n-1}^{0}=0$ is not characteristic and therefore for $\lambda$ the polynomial $\widetilde{p_{0}}\left(i \xi_{1}, \ldots, i \xi_{n-2}, \lambda, s\right)$ has exactly $2 m$ roots. In this case, if $\operatorname{Re} s>0$, the number of roots $\lambda_{j}\left(\xi_{1}, \ldots, \xi_{n-2}, s\right)$, with the multiplicity of the polynomial $\widetilde{p_{0}}\left(i \xi_{1}, \ldots, i \xi_{n-2}, \lambda, s\right)$ taken into account, will be equal to $m$ provided that $\operatorname{Re} \lambda_{j}<0$. Indeed, recalling that equation (1) is hyperbolic, the equation $\widetilde{p_{0}}\left(i \xi_{1}, \ldots, i \xi_{n-2}, \lambda, s\right)=0$ has no purely imaginary roots with respect to $\lambda$. Since the roots $\lambda_{j}$ are continuous functions of $s$, we can determine the number of roots $\lambda_{j}$ with $\operatorname{Re} \lambda_{j}<0$ by passing to the limits 
as $\operatorname{Re} s \rightarrow+\infty$. Since the equality

$$
\widetilde{p}_{0}\left(i \xi_{1}, \ldots, i \xi_{n-2} \lambda, s\right)=s^{2 m} \widetilde{p}_{0}\left(i \frac{\xi_{1}}{s}, \ldots, i \frac{\xi_{n-2}}{s}, \frac{\lambda}{s}, 1\right)
$$

holds, it is clear that the ratios $\lambda_{j} / s$, where $\lambda_{j}$ are the roots of the equation $\widetilde{p_{0}}\left(i \xi_{1}, \ldots, i \xi_{n-2}, \lambda, s\right)=0$, tend to the roots $\mu_{j}$ of the equation $\widetilde{p_{0}}(0, \ldots 0, \mu, 1)=0$ as $\operatorname{Re} s \rightarrow+\infty$. The latter roots are real and different because equation (1) is hyperbolic. If $s$ is taken positive and sufficiently large, then for $\mu_{j} \neq 0$ we have $\lambda_{j}=s \mu_{j}+o(s)$. But $\mu_{j} \neq 0$, since the plane $x_{n}^{0}=0$ is not characteristic. Therefore the number of roots $\lambda_{j}$ with $\operatorname{Re} \lambda_{j}<0$ coincides with the number of roots $\mu_{j}$ with $\mu_{j}<0$. Since the characteristic planes of equation (1), passing through the $(n-2)$-dimensional plane $x_{n}^{0}=x_{n-1}^{0}=0$, are determined by the equalities $\mu_{j} x_{n-1}^{0}+x_{n}^{0}=$ $0, j=1, \ldots, 2 m$, condition 2 implies that for $\operatorname{Re} \lambda_{j}<0$ the number of roots $\lambda_{j}$ is equal to $m$.

We give another equivalent description of the space $W_{\alpha}^{k}(D)$. On the unit sphere $S^{n-1}: x_{1}^{2}+\cdots+x_{n}^{2}=1$ choose a coordinate system $\left(\omega_{1}, \ldots, \omega_{n-1}\right)$ such that in the domain $D$ the transformation

$$
I: \tau=\log r, \omega_{j}=\omega_{j}\left(x_{1}, \ldots, x_{n}\right), \quad j=1, \ldots, n-1,
$$

is one-to-one, nondegenerate, and infinitely differentiable. Since the cone $\Gamma=\partial D$ is strictly convex at the point $O(0, \ldots, 0)$, such coordinates evidently exist. As a result of the above transformation, the domain $D$ will become the infinite cylinder $G$ bounded by the infinitely differentiable surface $\partial G=I\left(\Gamma^{\prime}\right)$.

Introduce the functional space $H_{\gamma}^{k}(G),-\infty<\gamma<\infty$, with the norm

$$
\|v\|_{H_{\gamma}^{k}(G)}^{2}=\sum_{i_{1}+j=0}^{k} \int_{G} e^{-2 \gamma \tau}\left\|\frac{\partial^{i_{1}+j} v}{\partial \tau^{i_{1}} \partial \omega^{j}}\right\|^{2} d \omega d \tau
$$

where

$$
\frac{\partial^{i_{1}+j} v}{\partial \tau^{i_{1}} \partial \omega^{j}}=\frac{\partial^{i_{1}+j} v}{\partial \tau^{i_{1}} \partial \omega_{1}^{j_{1}} \cdots \partial \omega_{n-1}^{j_{n-1}}}, \quad j=j_{1}+\cdots+j_{n-1} .
$$

As shown in [16], a function $u(x) \in W_{\alpha}^{k}(D)$ iff $\widetilde{u}=u\left(I^{-1}(\tau, \omega)\right) \in$ $H_{(\alpha+k)-\frac{n}{2}}^{k}(G)$, and the estimates

$$
c_{1}\|\widetilde{u}\|_{H_{(\alpha+k)-\frac{n}{2}}^{k}(G)} \leq\|u\|_{W_{\alpha}^{k}(D)} \leq c_{2}\|\widetilde{u}\|_{H_{(\alpha+k)-\frac{n}{2}}^{k}(G)}
$$

hold, where $I^{-1}$ is the inverse transformation of $I$ and the positive constants $c_{1}$ and $c_{2}$ do not depend on $u$.

It can be easily verified that the condition $v \in H_{\gamma}^{k}(G)$ is equivalent to the condition $e^{-\gamma \tau} v \in W^{k}(G)$, where $W^{k}(G)$ is the Sobolev space. Denote 
by $H_{\gamma}^{k}(\partial G)$ a set of $\psi$ such that $e^{-\gamma \tau} \psi \in W^{k}(\partial G)$, and by $W_{\alpha-\frac{1}{2}}^{k}(\Gamma)$ a set of all $\varphi$ for which $\widetilde{\varphi}=\varphi\left(I^{-1}(\tau, \omega)\right) \in H_{(\alpha+k)-\frac{n}{2}}^{k}(\partial G)$. Assume that

$$
\|\varphi\|_{W_{\alpha-\frac{1}{2}}^{k}(\Gamma)}=\|\widetilde{\varphi}\| H_{(\alpha+k)-\frac{n}{2}}^{k}(\partial G) .
$$

Spaces $W_{\alpha}^{k}(D)$ possess the following simple properties:

1) if $u \in W_{\alpha}^{k}(D)$, then $\frac{\partial^{i} u}{\partial x^{i}} \in W_{\alpha}^{k-i}(D), \quad 0 \leq i \leq k$;

2) $W_{\alpha-1}^{k+1}(D) \subset W_{\alpha}^{k}(D)$;

3) if $u \in W_{\alpha-1}^{k+1}(D)$, then by the well-known embedding theorems we have $\left.u\right|_{\Gamma} \in W_{\alpha-\frac{1}{2}}^{k}(\Gamma),\left.\frac{\partial^{i} u}{\partial \nu^{i}}\right|_{\Gamma^{\prime}} \in W_{\alpha-\frac{1}{2}}^{k-i}(\Gamma), i=1, \ldots, m-1$;

4) if $u \in W_{\alpha-1}^{k+1}(D)$, then $f=p(x, \partial) u \in W_{\alpha-1}^{k+1-2 m}(D)$.

In what follows we will need, in spaces $W_{\alpha}^{k}(D), W_{\alpha-\frac{1}{2}}^{k}(\Gamma)$, other norms depending on the parameter $\gamma=(\alpha+k)-\frac{n}{2}$ and equivalent to the original norms.

Set

$$
\begin{aligned}
& R_{\omega, \tau}^{n}=\left\{-\infty<\tau<\infty,-\infty<\omega_{i}<\infty, i=1, \ldots, n-1\right\}, \\
& R_{\omega, \tau,+}^{n}=\left\{(\omega, \tau) \in R_{\omega, \tau}^{n}: \omega_{n-1}>0\right\}, \omega^{\prime}=\left(\omega_{1}, \ldots, \omega_{n-2}\right), \\
& R_{\omega^{\prime}, \tau}^{n-1}=\left\{-\infty<\tau<\infty,-\infty<\omega_{i}<\infty, i=1, \ldots, n-2\right\} .
\end{aligned}
$$

Denote by $\widetilde{v}\left(\xi_{1}, \ldots, \xi_{n-2}, \xi_{n-1}, \xi_{n}-i \gamma\right)$ the Fourier transform of the function $e^{-\gamma \tau} v(\omega, \tau)$, i.e.,

$$
\begin{gathered}
\widetilde{v}\left(\xi_{1}, \ldots, \xi_{n-1}, \xi_{n}-i \gamma\right)=(2 \pi)^{-\frac{n}{2}} \int v(\omega, \tau) e^{-i \omega \xi^{\prime}-i \tau \xi_{n}-\gamma \tau} d \omega d \tau, \\
i=\sqrt{-1}, \quad \xi^{\prime}=\left(\xi_{1}, \ldots, \xi_{n-1}\right),
\end{gathered}
$$

and by $\widehat{v}\left(\xi, \ldots, \xi_{n-2}, \omega_{n-1}, \xi_{n}-i \gamma\right)$ the partial Fourier transform of the function $e^{-\gamma \tau} v(\omega, \tau)$ with respect to $\omega^{\prime}, \tau$.

We can introduce the following equivalent norms:

$$
\begin{gathered}
\|\| v\left\|\left.\right|_{R^{n}, k, \gamma} ^{2}=\int_{R^{n}}\left(\gamma^{2}+|\xi|^{2}\right)^{k}\right\| \widetilde{v}\left(\xi_{1}, \ldots, \xi_{n-1}, \xi_{n}-i \gamma\right) \|^{2} d \xi \\
\|\| v \mid \|_{R_{+}^{n}, k, \gamma}^{2}=\int_{0}^{\infty} \int_{R^{n-1}} \sum_{j=0}^{k}\left(\gamma^{2}+\left|\xi^{\prime}\right|^{2}\right)^{k-j} \times \\
\times\left\|\frac{\partial^{j}}{\partial \omega_{n-1}^{j}} \hat{v}\left(\xi_{1}, \ldots, \xi_{n-2}, \omega_{n-1}, \xi_{n}-i \gamma\right)\right\|^{2} d \xi^{\prime} d \omega_{n-1},
\end{gathered}
$$

in the above-considered spaces $H_{\gamma}^{k}\left(R_{\omega, \tau}^{n}\right)$ and $H_{\gamma}^{k}\left(R_{\omega, \tau,+}^{n}\right)$.

Let $\varphi_{1}, \ldots, \varphi_{N}$ be the partitioning of unity into $G^{\prime}=G \cap\{\tau=0\}$, where $G=I(D)$, i.e., $\sum_{j=1}^{N} \varphi_{j}(\omega) \equiv 1$ in $G^{\prime}, \varphi_{j} \in C^{\infty}\left(\bar{G}^{\prime}\right)$, the supports of 
functions $\varphi_{1}, \ldots, \varphi_{N-1}$ lie in the boundary half-neighborhoods, while the support of function $\varphi_{N}$ lies inside $G^{\prime}$. Then for $\gamma=(\alpha+k)-\frac{n}{2}$ the equalities

$$
\begin{gathered}
\|\| u\|\|_{G, k, \gamma}^{2}=\sum_{j=1}^{N-1}\left\||| \varphi_{j} u\right\|\left\|_{R_{+, k, \gamma}^{n}}^{2}+\right\|\left\|\varphi_{N} u \mid\right\|_{R^{n}, k, \gamma}^{2}, \\
\|\| u\left\|\left.\right|_{\partial G, k, \gamma} ^{2}=\sum_{j=1}^{N-1}\right\|\left|\varphi_{j} u\right| \|_{R_{\omega^{\prime}, \tau, k, \gamma}^{n-1}}^{2}
\end{gathered}
$$

define equivalent norms in the spaces $W_{\alpha}^{k}(D)$ and $W_{\alpha-\frac{1}{2}}^{k}(\Gamma)$, where the norms on the right sides of these equalities are taken in the terms of local coordinates [17].

First we assume that equation (1) contains only higher terms, i.e., $p(x, \xi) \equiv$ $p_{0}(\xi)$. Equation (1) and the boundary conditions (2) written in terms of the coordinates $\omega, \tau$ have the form

$$
\begin{gathered}
e^{-2 m \tau} A(\omega, \partial) u=f \\
\left.e^{-i \tau} B_{i}(\omega, \partial) u\right|_{\partial G}=g_{i}, \quad i=0, \ldots, m-1,
\end{gathered}
$$

or

$$
\begin{gathered}
A(\omega, \partial) u=\widetilde{f}, \\
\left.B_{i}(\omega, \partial) u\right|_{\partial G}=\widetilde{g}_{i}, \quad i=0, \ldots, m-1,
\end{gathered}
$$

where $A(\omega, \partial)$ and $B_{i}(\omega, \partial)$ are, respectively, the differential operators of orders $2 m$ and $i$, with infinitely differentiable coefficients depending only on $\omega$, while $\widetilde{f}=e^{2 m \tau} f$ and $\widetilde{g}_{i}=e^{i \tau} g_{i}, \quad i=0,1, \ldots, m-1$.

Thus, for the transformation $I: D \rightarrow G$, the unbounded operator $T$ of the problem (1), (2) transforms to the unbounded operator

$$
\widetilde{T}: H_{\gamma}^{k}(G) \rightarrow H_{\gamma}^{k+1-2 m}(G) \times \prod_{i=0}^{m-1} H_{\gamma}^{k-i}(\partial G)
$$

with the domain of definition $H_{\gamma}^{k+1}(G)$, acting by the formula

$$
\widetilde{T} u=\left(A(\omega, \partial) u,\left.B_{0}(\omega, \partial) u\right|_{\partial G}, \ldots,\left.B_{m-1}(\omega, \partial) u\right|_{\partial G}\right)
$$

where $\gamma=(\alpha+k)-\frac{n}{2}$. Note that written in terms of the coordinates $\omega, \tau$ the functions $f=(\omega, \tau) \in H_{\gamma-2 m}^{k+1-2 m}(G), g_{i}(\omega, \tau) \in H_{\gamma-i}^{k-i}(\partial G), i=$ $0, \ldots, m-1$, if $f(x) \in W_{\alpha-1}^{k+1-2 m}(D), g_{i}(x) \in W_{\alpha-\frac{1}{2}}^{k-i}(\Gamma), i=0, \ldots, m-1$. Therefore the functions $\widetilde{f}=e^{2 m \tau} f \in H_{\gamma}^{k+1-2 m}(G), \widetilde{g}_{i}=e^{i \tau} g_{i} \in H_{\gamma}^{k-i}(\partial G)$, $i=0, \ldots, m-1$. 
Since by condition 1 each generatrix of the cone $\Gamma$ has the direction of a spatial-type normal, due to the convexity of $K_{m}$ each beam coming from the vertex $O$ into the conic domain $D$ also has the direction of a spatialtype normal. Therefore equation (4) is strictly hyperbolic with respect the $\tau$-axis. It was shown above that the fulfillment of condition 1 implies the fulfillment of condition 4. Therefore, according to the results of [17],

for $\gamma \geq \gamma_{0}$, where $\gamma_{0}$ is a sufficiently large number, the operator $\overline{\widetilde{T}}$ has the bounded right inverse operator $\overline{\widetilde{T}}^{-1}$. Thus for any $\widetilde{f} \in H_{\gamma}^{k+1-2 m}(G)$, $\tilde{g}_{i} \in H_{\gamma}^{k-i}(\partial G), i=0, \ldots, m-1$, when $\gamma \geq \gamma_{0}$, the problem (5), (6) is uniquely solvable in the space $H_{\gamma}^{k}(G)$, and for the solution $u$ we have the estimate

$$
\||| u\|_{G, k, \gamma}^{2} \leq C\left(\sum_{i=0}^{m-1}\left\|\left|\widetilde{g}_{i}\right|\right\|_{\partial G, k-i, \gamma}+\frac{1}{\gamma} \mid\|\widetilde{f}\|_{G, k+1-2 m, \gamma}\right)
$$

with the positive constant $C$ not depending on $\gamma, f$ and $\widetilde{g}_{i}, i=0, \ldots, m-1$.

Hence it immediately follows that the theorem and the estimate (3) are valid in the case $p(x, \xi) \equiv p_{0}(\xi)$.

Remark. The estimate (7) with the coefficient $\frac{1}{\gamma}$ at $\|\widetilde{f}\| \|_{G, k+1-2 m, \gamma}$, obtained in the appropriately chosen norms (4), enables one to prove the theorem also when equation (1) contains lower terms, since the latter give arbitrarily small perturbations for sufficiently large $\gamma$.

\section{REFERENCES}

1. A.V.Bitsadze, Some classes of partial differential equations. (Russian) Nauka, Moscow, 1981.

2. S.L.Sobolev, Some applications of functional analysis in mathematical physics. (Russian) Publ. Sib. Otd. Akad. Nauk SSSR, Novosibirsk, 1962.

3. R.Courant, Partial differential equations. New York-London, 1962.

4. M.Riesz, L'integrale de Riemann-Liouville et le problem de Cauchy. Acta Math. 81(1949), 107-125.

5. L.Lundberg, The Klein-Gordon equation with light-cone data. Commun. Math. Phys. 62(1978), No. 2, 107-118.

6. A.A.Borgardt and D.A.Karnenko, The characteristic problem for the wave equation with mass. (Russian) Differentsial'nye Uravneniya 20(1984), No. 2, 302-308.

7. S.L.Sobolev, Some new problems of the theory of partial differential equations of hyperbolic type. (Russian) Mat. Sb. 11(53)(1942), No. 3, 155-200. 
8. A.V.Bitsadze, On mixed type equations on three-dimensional domains. (Russian) Dokl. Akad. Nauk SSSR 143(1962), No. 5, 1017-1019.

9. A.M.Nakhushev, A multidimensional analogy of the Darboux problem for hyperbolic equations. (Russian) Dokl. Akad. Nauk SSSR 194(1970), No. 1, 31-34.

10. T.Sh.Kalmenov, On multidimensional regular boundary value problems for the wave equation. (Russian) Izv. Akad. Nauk Kazakh. SSR. Ser. Fiz.-Mat. (1982), No. 3, 18-25.

11. A.A.Dezin, Invariant hyperbolic systems and the Goursat problem. (Russian) Dokl. Akad. Nauk SSSR 135(1960), No. 5, 1042-1045.

12. F.Cagnac, Probleme de Cauchy sur la conoide caracteristique. Ann. Mat. Pure Appl. 104(1975), 355-393.

13. J.Tolen, Probléme de Cauchy sur la deux hypersurfaces caracteristiques sécantes. C.R. Acad. Sci. Paris Sér. A-B 291(1980), No. 1, A49-A52.

14. S.S.Kharibegashvili, The Goursat problems for some class of hyperbolic systems. (Russian) Differentsial'nye Uravneniya 17(1981), No. 1. 157-164.

15. — - On a multidimensional problem of Goursat type for second order strictly hyperbolic systems. (Russian) Bull. Acad. Sci. Georgian SSR 117(1985), No. 1, 37-40.

16. V.A.Kondratyev, Boundary value problems for elliptic equations in domains with conic or corner points. (Russian) Trudy Moskov. Mat. Obshch. 16(1967), 209-292.

17. M.S.Agranovich, Boundary value problems for systems with a parameter. (Russian) Mat. Sb. 84(126)(1971), No. 1, 27-65.

(Received 25.12.1992)

Author's address:

I.Vekua Institute of Applied Mathematics

of Tbilisi State University

2 University St., 380043 Tbilisi

Republic of Georgia 